\title{
CONVERGENCE OF AN ENTROPIC SEMI-DISCRETIZATION FOR NONLINEAR FOKKER-PLANCK EQUATIONS IN $\mathbb{R}^{d}$
}

\author{
J. A. Carrillo, M. P. Gualdani, and A. Jüngel
}

\begin{abstract}
A nonlinear degenerate Fokker-Planck equation in the whole space is analyzed. The existence of solutions to the corresponding implicit Euler scheme is proved, and it is shown that the semidiscrete solution converges to a solution of the continuous problem. Furthermore, the discrete entropy decays monotonically in time and the solution to the continuous problem is unique. The nonlinearity is assumed to be of porous-medium type. For the (given) potential, either a less than quadratic growth condition at infinity is supposed or the initial datum is assumed to be compactly supported. The existence proof is based on regularization and maximum principle arguments. Upper bounds for the tail behavior in space at infinity are also derived in the at-most-quadratic growth case.
\end{abstract}

\section{Introduction}

Fokker-Planck equations play an important role in applications, for instance in semiconductor theory $[\mathbf{1 5}],[\mathbf{1 7}]$, plasma physics $[\mathbf{2 0}]$, population dynamics $[\mathbf{3}]$, stellar dynamics $[\mathbf{1 2}]$, vortex dynamics $[\mathbf{8}]$ and several other applications in mathematical physics [13]. We analyze in this paper the nonlinear Fokker-Planck equation

$$
\partial_{t} n=\operatorname{div}(\nabla f(n)+n \nabla V) \quad \text { in } \mathbb{R}^{d}, 0<t<T,
$$

with initial conditions

$$
n(\cdot, 0)=n_{I} \geq 0 \quad \text { in } \mathbb{R}^{d} .
$$

In most applications, the function $n=n(x, t)$ denotes a density such that it should hold $n \geq 0$ in $\mathbb{R}^{d}$.

2000 Mathematics Subject Classification. 35K65, 35B40.

Key words. Fokker-Planck equation, drift-diffusion equation, degenerate parabolic equation, existence of weak solutions, uniqueness of solutions, nonnegativity, implicit Euler scheme, relative entropy. 
In the absence of the potential $V(x)$, we obtain the nonlinear filtration equations with the widely studied porous medium and fast diffusion equations as particular cases, e.g. [2], [23], [24]. When the potential is coupled to the density $n$ via the Poisson equation, we obtain the nonlinear drift-diffusion model, which has been employed, in particular with $f(s)=s^{5 / 3}$, in semiconductor theory [11], [14]. The case of linear diffusion $f(s)=s$ arises, for instance, in semiconductor modeling [15] or large-population dynamics [18]. Nonlinear Fokker-Planck models have been proposed for population dynamics in [3].

When the nonlinear Fokker-Planck (or drift-diffusion) equation is considered in a bounded domain, appropriate no-flux boundary conditions are imposed in order to conserve the total mass of the density $n$. The existence and long time behavior of weak solutions for (1)-(2) in bounded domains and in the whole space have been studied in $[\mathbf{6}]$ in full generality following procedures introduced in [19]. By approximation procedures starting from non-degenerate diffusions in bounded domains with noflux boundary conditions [3], the authors obtain the existence of global weak solutions in two different situations for the potential $V(x)$ : either its Laplacian is bounded or general growth at infinity of the potential is allowed but with compactly supported initial data. Moreover, the asymptotic behavior of these solutions is proved to be given by stationary solutions of this problem with an exponential relaxation speed under the assumption of uniformly convex potentials. On the other hand, the long-time behavior of whole-space solutions to (1), coupled to a Poisson equation, was analyzed in [4] for smooth solutions. Furthermore, the stability of (1) with linear diffusion in $\mathbb{R}^{d}$ was treated in [18].

The goal of this paper is to show that the implicit Euler semi-discretization of (1) in the whole space $\mathbb{R}^{d}$ is globally solvable in time under slightly improved assumptions on the initial data and on the potential with respect to $[\mathbf{6}]$. We also show the convergence of this semi-discrete scheme towards global weak solutions of (1), recovering in this way the results in $[6]$, and prove the uniqueness of solutions to the limit problem (1). Furthermore, we show that the approximation keeps the exponential relaxation towards equilibrium under the assumption of uniformly convex potentials. Here, an important ingredient will be the generalized logarithmic Sobolev inequalities shown in $[\mathbf{6}]$ and later generalized to other situations in $[\mathbf{7}]$, see also $[\mathbf{1}],[\mathbf{2 5}]$. The formal use of these inequalities to show the exponential convergence towards equilibrium for the implicit Euler discretization was already reported in [5]. 
More precisely, we deal with

$$
\frac{1}{\tau}\left(n_{k}-n_{k-1}\right)=\operatorname{div}\left(\nabla f\left(n_{k}\right)+n_{k} \nabla V\right) \quad \text { in } \mathbb{R}^{d}, t \in\left(t_{k-1}, t_{k}\right],
$$

where $0=t_{0}<\cdots<t_{N}=T$ is a partition of $[0, T]$ with $t_{k}=k T / N, \tau=$ $T / N$, and $n_{0}=n_{I}$. We prove that this scheme is well-defined, convergent, and entropy-decaying, i.e., the semi-discrete solution $n_{k}$ converges to a (weak nonnegative) solution of the continuous problem (1)-(2) and the discrete entropy

$$
E\left(n_{k}\right)=\int_{\mathbb{R}^{d}}\left(H\left(n_{k}\right)+n_{k} V\right) d x
$$

is nonincreasing. Here, $H(s)$ is a primitive of

$$
h(s)=\int_{1}^{s} \frac{f^{\prime}(\sigma)}{\sigma} d \sigma, \quad s \geq 0 .
$$

In this way, the convergence result also provides an existence proof for (1)-(2). When the potential $V(x)$ is uniformly convex, the relative entropy is shown to satisfy the inequality

$$
E\left(n_{k}\right)-E\left(n_{\infty}\right) \leq(1+2 \tau \lambda)^{-k}\left(E\left(n_{I}\right)-E\left(n_{\infty}\right)\right), \quad k \in \mathbb{N},
$$

where $n_{\infty}$ is the solution of the equilibrium state $\nabla f\left(n_{\infty}\right)+n_{\infty} \nabla V=0$ with the same mass as $n_{I}$, and $\lambda>0$ measures the convexity of the potential $V$ in the sense of $\xi^{\top} \operatorname{Hess}(V(x)) \xi \geq \lambda|\xi|^{2}$, where $\operatorname{Hess}(V(x))$ denotes the Hessian of $V(x)$.

Our first main assumption is that the nonlinearity is degenerate and given by $f(s)=s^{\alpha}$ with $\alpha>1$. We can also allow for more general nonlinearities with a similar behavior, see Remark 2.1. The second assumption concerns the potential $V$. We assume that either the potential grows at most quadratically (in the sense of assumption (A2) below) or the potential is locally an $H^{1}$ function with arbitrary growth at infinity but the initial datum is compactly supported (see assumption (A2') below).

The originality of this paper with respect to the previous literature consists of the following facts. We first provide a different proof of the existence of solutions to the nonlinear Fokker-Planck equation in the whole space based on the implicit Euler scheme. We are able to give an upper bound for the solution with the same decay at infinity as the initial datum. More precisely, under the assumption (A2) below, if $n_{I}(x) \leq$ $C|x|^{-\gamma}$ for all $|x| \geq R_{0}\left(C, \gamma\right.$ and $R_{0}$ are some positive constants), then $n$ is bounded from above by a function of the type $|x|^{-\gamma}$ for all $|x| \geq R$, where $R \geq R_{0}$. Finally, the semi-discretization keeps the important 
entropy decay and its rate characterizing the long-time asymptotics of the solutions.

The paper is organized as follows. In Section 2 the assumptions and main results are presented and discussed. Section 3 is concerned with the existence of solutions to the discrete problem (3) and the continuous problem (1)-(2) under assumption (A2). The existence of solutions to both problems under assumption (A2') is proved in Section 3.4. In Section 4 we show the uniqueness of weak solutions to (1)-(2) assuming $W^{2, \infty}$ potentials. Finally, the discrete entropy decay is shown in Section 5 .

\section{Assumptions and main results}

We impose the following assumptions:

(A1) Nonlinearity: We assume the typical degenerate diffusion case $f(s)=s^{\alpha}$ with $\alpha>1$.

(A2) Potential and initial datum:

(a) The potential $V \in H_{\text {loc }}^{2}\left(\mathbb{R}^{d}\right)$ is a nonnegative function with $\Delta V \in L^{\infty}\left(\mathbb{R}^{d}\right)$ and there exists a constant $c_{0}>0$ such that

$$
\frac{x \cdot \nabla V(x)}{|x|^{2}} \geq c_{0}>0 .
$$

(b) The initial datum $n_{I} \in L^{1}\left(\mathbb{R}^{d}\right) \cap L^{\infty}\left(\mathbb{R}^{d}\right)$ is nonnegative and there exist constants $c_{1}>0$ and $R_{0}>0$ such that for all $|x| \geq R_{0}$, $n_{I}(x) \leq c_{1}\left(R_{0} /|x|\right)^{\gamma}$, where $\gamma>\max \left\{\|\Delta V\|_{L^{\infty}\left(\mathbb{R}^{d}\right)} / c_{0},(d+\right.$ 2)/2\}.

(A2') Potential and initial datum:

(a) The potential $V \in H_{\text {loc }}^{2}\left(\mathbb{R}^{d}\right)$ is a nonnegative function such that $V(x) \rightarrow+\infty$ as $|x| \rightarrow+\infty$.

(b) The initial datum $0 \leq n_{I} \in L^{\infty}\left(\mathbb{R}^{d}\right)$ is compactly supported.

Remark 2.1 (Generalizations). Assumption (A1) can be relaxed. Our proof also works if $f:[0, \infty) \rightarrow[0, \infty)$ is a strictly increasing $C^{2}$ function such that $f(0)=0, s \mapsto \gamma s f^{\prime \prime}(s)+(\gamma+2-d) f^{\prime}(s)$ is nondecreasing, and $f^{-1}$ is Hölder continuous of order $\theta \in(0,1)$. Moreover, $h$, defined in (5), is assumed to be an $L_{\text {loc }}^{1}$ function on $[0, \infty)$ with the properties $h(0)>-\infty$ and $h(\infty)=\infty$. Finally, we need that $x \mapsto f\left(|x|^{-\kappa}\right)^{2}$ and $x \mapsto H\left(|x|^{-\kappa}\right)$ are integrable near infinity for some $\kappa>2$ in the assumption (A2b).

Remark 2.2 (Hypotheses on the potential). Assumption (A2) is satisfied by functions $V$ which grow at most quadratically (in the sense that 
$V(x)=V_{0}(|x|)$ and $V_{0}$ satisfies $\left|V_{0}^{\prime}(\rho)\right| \leq k_{1} \rho$ for all $\rho \geq 0$ and for some $\left.k_{1}>0\right)$. For instance, confining potentials of the form $V(x)=$ $|x|^{2}+c_{1}|x|+c_{2}$, with $c_{1}, c_{2} \geq 0$, are admissible. On the other hand, potentials which grow faster than quadratically, e.g. $V(x)=|x|^{4}$, are allowed in assumption (A2'). Here, we need to restrict the initial data to compactly supported functions.

Remark 2.3 (Hypotheses on the potential). Condition (6) is not needed to prove the existence of a constant upper bound for the solutions, used in the existence proof. However, we will employ it to show that the upper bound decays as $|x|^{-\gamma}$ at infinity if we assume so for the initial data under assumptions (A2). It is an open problem to generalize such a result in case the potential verifies assumptions (A2').

Our main results are as follows.

Theorem 2.1 (Well-Posedness of the Implicit Euler Scheme). Let the assumptions (A1) and (A2) or (A2') hold and let $k \in\{1, \ldots, N\}$. If (A2) holds then assume that $0<\tau<1 /\|\Delta V\|_{L^{\infty}\left(\mathbb{R}^{d}\right)}$. Then there exists a weak solution $0 \leq n_{k} \in L^{1}\left(\mathbb{R}^{d}\right) \cap L^{\infty}\left(\mathbb{R}^{d}\right)$ to (3) satisfying $f\left(n_{k}\right)=$ $n_{k}^{\alpha} \in H^{1}\left(\mathbb{R}^{d}\right)$ and

$$
\int_{\mathbb{R}^{d}}\left(\nabla f\left(n_{k}\right)+n_{k} \nabla V\right) \cdot \nabla \psi d x=-\frac{1}{\tau} \int_{\mathbb{R}^{d}}\left(n_{k}-n_{k-1}\right) \psi d x
$$

for all compactly supported $0 \leq \psi \in H^{1}\left(\mathbb{R}^{d}\right)$. Furthermore, under the assumption (A2), $n_{k}$ satisfies

$$
n_{k} \leq \bar{n}_{k}:=\frac{c_{2}}{\left(1-\tau\|\Delta V\|_{\left.L^{\infty}\left(\mathbb{R}^{d}\right)\right)^{k}}\right.} \max \left\{1,\left(\frac{R}{|x|}\right)^{\gamma}\right\} \quad \text { in } \mathbb{R}^{d},
$$

where $c_{2}=\max \left\{\left\|n_{I}\right\|_{L^{\infty}\left(B_{R_{0}}\right)}, c_{1}\right\}$.

Theorem 2.2 (Convergence of the Semi-discretization). Let the assumptions (A1) and (A2) or (A2') hold and let $T>0$. Then there exists a weak solution $0 \leq n \in L^{\infty}\left(0, T ; L^{1}\left(\mathbb{R}^{d}\right)\right) \cap L^{\infty}\left(0, T ; L^{\infty}\left(\mathbb{R}^{d}\right)\right)$ to $(1)-(2)$ satisfying $\partial_{t} n \in L^{\overline{2}}\left(0, T ; H^{-1}\left(\mathbb{R}^{d}\right)\right), f(n)=n^{\alpha} \in L^{2}\left(0, T ; H^{1}\left(\mathbb{R}^{d}\right)\right)$, and

$$
\int_{0}^{T}\left\langle\partial_{t} n, \psi\right\rangle_{H^{-1}, H^{1}} d t=-\int_{0}^{T} \int_{\mathbb{R}^{d}}(\nabla f(n)+n \nabla V) \cdot \nabla \psi d x d t
$$

for all $\psi \in C_{0}^{\infty}\left(\mathbb{R}^{d} \times[0, T]\right)$, where $C_{0}^{\infty}$ is the space of all $C^{\infty}$ functions with compact support. Furthermore, under the assumption (A2), $n$ satisfies

$$
n \leq \bar{n}:=c_{2} e^{T\|\Delta V\|_{L^{\infty}\left(\mathbb{R}^{d}\right)} \max }\left\{1,\left(\frac{R}{|x|}\right)^{\gamma}\right\} \quad \text { in } \mathbb{R}^{d},
$$

where $c_{2}$ is defined in Theorem 2.1. 
For the following theorem we define the equilibrium function $n_{\infty}$ which is a solution to $\nabla f\left(n_{\infty}\right)+n_{\infty} \nabla V=0$ inside its support and $\int_{\mathbb{R}^{d}} n_{\infty} d x=\int_{\mathbb{R}^{d}} n_{I} d x$. This means that $n_{\infty}(x)=\underline{h}^{-1}(K-V(x))$, $x \in \mathbb{R}^{d}$, where $h(s)$ is given in (5), $\underline{h}^{-1}$ is the generalized inverse

$$
\underline{h}^{-1}: \mathbb{R} \rightarrow[0, \infty], \quad \underline{h}^{-1}(\sigma)=\left\{\begin{array}{l}
0: \sigma \leq h(0+) \\
h^{-1}(\sigma): h(0+)<\sigma<\infty,
\end{array}\right.
$$

and $K \in \mathbb{R}$ is uniquely determined by the conservation of mass

$$
\int_{\mathbb{R}^{d}} n_{\infty} d x=\int_{\mathbb{R}^{d}} n_{I} d x .
$$

Notice that $n_{\infty}$ is compactly supported since $V(x) \rightarrow \infty$ as $x \rightarrow \infty$ and $h(0)>-\infty$. We refer to [6, Subsection 3.1] for more details on the equilibrium solution properties.

Theorem 2.3 (Exponential Relaxation of the Entropy). Let the assumptions (A1) and (A2) or (A2') hold and suppose that $V$ is uniformly convex, i.e., there exists $\lambda>0$ such that for all $\xi, x \in \mathbb{R}^{d}$,

$$
\xi^{\top} \operatorname{Hess}(V(x)) \xi \geq \lambda|\xi|^{2} .
$$

Furthermore, let $n_{k}$ be the recursively defined sequence of solutions to (3) according to Theorem 2.1. Then, for all $k=1, \ldots, N$,

$$
E\left(n_{k}\right)-E\left(n_{\infty}\right) \leq(1+2 \lambda \tau)^{-k}\left(E\left(n_{I}\right)-E\left(n_{\infty}\right)\right) .
$$

As a consequence of the previous theorem by passing to the limit in the discretization, one can recover also the exponential decay of the entropy for the initial value problem (1)-(3) as proven in [6, Theorem 16]. Let us remark that all the above results can be also obtained in general smooth bounded domains with no-flux boundary conditions.

Theorem 2.4 (Uniqueness of solutions). Let the assumptions (A1) and (A2) or (A2') hold and let $T>0$. Furthermore, we assume that $V \in$ $W^{2, \infty}\left(\mathbb{R}^{d}\right)$. Then there exists a unique weak solution in the sense of Theorem 2.2.

The uniqueness proof is based on the $H^{-1}$ technique [10], which is the standard method to prove the uniqueness of solutions to degenerate problems. The main difficulty is the drift term which is treated as in $[\mathbf{2 1}]$. 


\section{Existence of solutions}

\subsection{Some auxiliary results.}

We first need to show some auxiliary results concerning a comparison principle between weak solutions and weak super-solutions for suitable regularizations of equation (1). In order to prove Theorem 2.1, we consider the following regularized problem:

$$
\begin{array}{ll}
\frac{1}{\tau}\left(n_{k}-n_{k-1}\right)=\operatorname{div}\left(\nabla f_{\varepsilon}\left(n_{k}\right)+n_{k} \nabla V\right) & \text { in } B_{m}, \\
\frac{\partial}{\partial \nu} f_{\varepsilon}\left(n_{k}\right)+n_{k} \frac{\partial V}{\partial \nu}=0 & \text { on } \partial B_{m}
\end{array}
$$

for given $n_{0}=n_{I} \geq 0$, where $\varepsilon \in(0,1), f_{\varepsilon}(s)=s^{\alpha}+\varepsilon s(s \geq 0)$, $B_{m}:=\{|x| \leq m\}$ and $\nu$ is the outward normal unit vector on $\partial B_{m}$. As the original equation is of degenerate type, the introduction of $f_{\varepsilon}$ makes the problem uniformly elliptic.

Lemma 3.1 (Comparison Principle). For given nonnegative functions $n_{0}, \hat{n}_{0} \in L^{\infty}\left(B_{m}\right)$, let $n_{k}, \hat{n}_{k}$, respectively, be weak solutions to

$$
\begin{aligned}
& \frac{1}{\tau}\left(n_{k}-n_{k-1}\right)=\operatorname{div}\left(\nabla f_{\varepsilon}\left(n_{k}\right)+n_{k} \nabla V\right), \\
& \frac{1}{\tau}\left(\hat{n}_{k}-\hat{n}_{k-1}\right) \geq \operatorname{div}\left(\nabla f_{\varepsilon}\left(\hat{n}_{k}\right)+\hat{n}_{k} \nabla V\right) \quad \text { in } B_{m},
\end{aligned}
$$

where $k \in\{1, \ldots, N\}$. Then it holds

$$
\int_{B_{m}}\left(n_{k}-\hat{n}_{k}\right)^{+} d x \leq \int_{B_{m}}\left(n_{k-1}-\hat{n}_{k-1}\right)^{+} d x,
$$

where $s^{+}=\max \{0, s\}$.

Proof: For the sake of simplicity, we set $n=n_{k}$ and $\hat{n}=\hat{n}_{k}$. We wish to use a regularized version of the test function $\operatorname{sign}^{+}\left(f_{\varepsilon}(n)-f_{\varepsilon}(\hat{n})\right)$ in the weak formulation of the above problems, where $\operatorname{sign}^{+}(s)=1$ if $s>0$ and $\operatorname{sign}^{+}(s)=0$ if $s \leq 0$. The positive sign function is approximated by

$$
\operatorname{sign}_{\omega}^{+}(s)= \begin{cases}1 & \text { if } s \geq \omega \\ 0 & \text { if } s \leq 0 \\ \frac{e+1}{e-1}\left(\frac{2 e^{s / \omega}}{e^{s / \omega}+1}-1\right) & \text { if } 0<s<\omega\end{cases}
$$

where $\omega>0$. Then, for any $s \in \mathbb{R}, \operatorname{sign}_{\omega}^{+}(s) \rightarrow \operatorname{sign}^{+}(s)$ as $\omega \rightarrow 0$. Moreover, $\left(\operatorname{sign}_{\omega}^{+}\right)^{\prime}(s)=2 e^{s / \omega} /\left(\omega\left(e^{s / \omega}+1\right)^{2}\right)$ for all $0<s<\omega$ and 
$\left(\operatorname{sign}_{\omega}^{+}\right)^{\prime}(s)=0$ for all $s<0$ and $s>\omega$ and thus, it holds $s\left(\operatorname{sign}_{\omega}^{+}\right)^{\prime}(s) \rightarrow$ 0 as $\omega \rightarrow 0$ for all $s \in \mathbb{R}$.

Now, we use $\psi=S_{\omega} \in H^{1}\left(B_{m}\right)$ with $S_{\omega}=\operatorname{sign}_{\omega}^{+}\left(f_{\varepsilon}(n)-f_{\varepsilon}(\hat{n})\right)$ as a test function in the weak formulations of $n$ and $\hat{n}$, respectively, and take the difference of both equations:

$$
\begin{aligned}
\frac{1}{\tau} \int_{B_{m}}(n-\hat{n}) S_{\omega} d x & -\frac{1}{\tau} \int_{B_{m}}\left(n_{k-1}-\hat{n}_{k-1}\right) S_{\omega} d x \\
\leq & -\int_{\mathbb{R}^{d}}(n-\hat{n}) \nabla V \cdot \nabla\left(f_{\varepsilon}(n)-f_{\varepsilon}(\hat{n})\right) S_{\omega}^{\prime} d x \\
& -\int_{\mathbb{R}^{d}}\left|\nabla\left(f_{\varepsilon}(n)-f_{\varepsilon}(\hat{n})\right)\right|^{2} S_{\omega}^{\prime} d x \\
= & I_{1}+I_{2} .
\end{aligned}
$$

We denote by $S_{\omega}^{\prime}$ the derivative of the function $\operatorname{sign}_{\omega}^{+}(\cdot)$ with respect to its argument. Since $S_{\omega}^{\prime} \geq 0$, we have $I_{2} \leq 0$. In order to treat $I_{1}$, we observe that a computation leads to

$$
0 \leq(n-\hat{n}) S_{\omega}^{\prime} \leq \frac{c(n-\hat{n})}{f(n)-f(\hat{n})+\varepsilon(n-\hat{n})} \leq \frac{c}{\varepsilon},
$$

where $c>0$ is a constant which is independent of $\omega$ (and $\varepsilon$ ). Moreover, $(n-\hat{n}) S_{\omega}^{\prime}$ converges pointwise a.e. to zero as $\omega \rightarrow 0$. Thus, applying Lebesgue's convergence theorem, we obtain

$$
\lim _{\omega \rightarrow 0} I_{1}=-\int_{B_{m}} \lim _{\omega \rightarrow 0}\left((n-\hat{n}) S_{\omega}^{\prime}\right) \nabla V \cdot \nabla\left(f_{\varepsilon}(n)-f_{\varepsilon}(\hat{n})\right) d x=0 .
$$

Passing to the limit $\omega \rightarrow 0$ in (15) gives

$$
\begin{aligned}
& \int_{B_{m}}(n-\hat{n}) \operatorname{sign}^{+}\left(f_{\varepsilon}(n)-f_{\varepsilon}(\hat{n})\right) d x \\
& \leq \int_{B_{m}}\left(n_{k-1}-\hat{n}_{k-1}\right) \operatorname{sign}^{+}\left(f_{\varepsilon}(n)-f_{\varepsilon}(\hat{n})\right) d x
\end{aligned}
$$

Since

$$
\begin{gathered}
\int_{B_{m}}(n-\hat{n}) \operatorname{sign}^{+}\left(f_{\varepsilon}(n)-f_{\varepsilon}(\hat{n})\right) d x=\int_{B_{m}}(n-\hat{n})^{+} d x \\
\int_{B_{m}}\left(n_{k-1}-\hat{n}_{k-1}\right) \operatorname{sign}^{+}\left(f_{\varepsilon}(n)-f_{\varepsilon}(\hat{n})\right) d x \leq \int_{B_{m}}\left(n_{k-1}-\hat{n}_{k-1}\right)^{+} d x
\end{gathered}
$$

the assertion follows.

Let us obtain some pointwise a-priori estimates for weak solutions of the problem (12). 
Lemma 3.2 (Supersolution). Let (A2) hold. Suppose that

$\tau<1 /\|\Delta V\|_{L^{\infty}\left(\mathbb{R}^{d}\right)}$ and let $k \in\{1, \ldots, N\}$. Furthermore, let $R>0$ such that (18) is satisfied (see below) and assume that $n_{k-1} \leq \bar{n}_{k-1}$. Then $\bar{n}_{k}$, defined in (8), is a solution to the variational inequality

$$
\text { (16) } \frac{1}{\tau} \int_{B_{m}}\left(\bar{n}_{k}-n_{k-1}\right) \psi d x+\int_{B_{m}}\left(\nabla f_{\varepsilon}\left(\bar{n}_{k}\right)+\bar{n}_{k} \nabla V\right) \cdot \nabla \psi d x \geq 0
$$

for all $0 \leq \psi \in H^{1}\left(B_{m}\right)$.

Proof: In the following, we write $\bar{n}$ instead of $\bar{n}_{k}$. We wish to perform an integration by parts in the left-hand side of (16). Since $\Delta \bar{n}$ is not defined on $|x|=R$, we have to split the domain of integration. For this, let $B_{R}=\{|x|<R\}$ for some $R>0$ which is specified in (18) and $m$ large enough such that $m>R$. We denote the left-hand side of (16) by $A$. Then, for all test functions $\psi \geq 0$, since $\bar{n}$ is constant on $B_{R}$ and $\bar{n}-n_{k-1} \geq 0$ in $B_{m}$

$$
\begin{aligned}
A= & \int_{B_{R}}\left(\frac{1}{\tau}\left(\bar{n}-n_{k-1}\right) \psi+\bar{n} \nabla V \cdot \nabla \psi\right) d x \\
& +\int_{\{R \leq|x| \leq m\}}\left(\frac{1}{\tau}\left(\bar{n}-n_{k-1}\right) \psi+\left(\nabla f_{\varepsilon}(\bar{n})+\bar{n} \nabla V\right) \cdot \nabla \psi\right) d x \\
\geq & \int_{B_{R}}\left(\frac{1}{\tau}\left(\bar{n}-n_{k-1}\right) \psi+\bar{n} \nabla V \cdot \nabla \psi\right) d x \\
& +\int_{\{R \leq|x| \leq m\}}\left(\nabla f_{\varepsilon}(\bar{n})+\bar{n} \nabla V\right) \cdot \nabla \psi d x .
\end{aligned}
$$

Integration by parts in both integrals leads to

$$
\begin{aligned}
A \geq & \int_{B_{R}}\left(\frac{1}{\tau}\left(\bar{n}-n_{k-1}\right)-\bar{n} \Delta V\right) \psi d x+\int_{\partial B_{R}} \bar{n} \frac{\partial V}{\partial \nu^{+}} \psi d s \\
& -\int_{\{R \leq|x| \leq m\}}\left(\Delta f_{\varepsilon}(\bar{n})+\nabla \bar{n} \cdot \nabla V+\bar{n} \Delta V\right) \psi d x \\
& +\int_{\partial B_{R}}\left(\frac{\partial}{\partial \nu^{-}} f_{\varepsilon}(\bar{n})+\bar{n} \frac{\partial V}{\partial \nu^{-}}\right) \psi d s \\
& +\int_{\partial B_{m}}\left(\frac{\partial}{\partial \nu^{+}} f_{\varepsilon}(\bar{n})+\bar{n} \frac{\partial V}{\partial \nu^{+}}\right) \psi d s,
\end{aligned}
$$


where $\nu^{+}=x /|x|$ is the exterior unit normal vector on $\partial B_{R}$ and $\partial B_{m}$, $\nu^{-}=-\nu^{+}$and $d s$ the surface measure. Since $\partial V / \partial \nu^{+}=-\partial V / \partial \nu^{-}$, the contributions $\bar{n} \partial V / \partial \nu^{ \pm}$cancel. Furthermore, we have on $\partial B_{R}$,

$$
\frac{\partial}{\partial \nu^{-}} f_{\varepsilon}(\bar{n})=-\nabla(f(\bar{n})+\varepsilon \bar{n}) \cdot \frac{x}{|x|}=2 \gamma \bar{n}\left(f^{\prime}(\bar{n})+\varepsilon\right) \frac{x}{|x|^{2}} \cdot \frac{x}{|x|} \geq 0,
$$

and on $\partial B_{m}$

$$
\frac{\partial}{\partial \nu^{+}} f_{\varepsilon}(\bar{n})+\bar{n} \frac{\partial V}{\partial \nu^{+}} \geq 0
$$

Hence, we obtain

$$
\begin{aligned}
A \geq & \int_{B_{R}}\left(\frac{1}{\tau}\left(\bar{n}-n_{k-1}\right)-\bar{n} \Delta V\right) \psi d x \\
& -\int_{\{R \leq|x| \leq m\}}\left(\Delta f_{\varepsilon}(\bar{n})+\nabla \bar{n} \cdot \nabla V+\bar{n} \Delta V\right) \psi d x \\
\geq & \frac{1}{\tau} \int_{B_{R}}\left(\left(1-\tau\|\Delta V\|_{L^{\infty}\left(\mathbb{R}^{d}\right)}\right) \bar{n}-n_{k-1}\right) d x \\
& -\int_{\{R \leq|x| \leq m\}}\left(\Delta f_{\varepsilon}(\bar{n})+\nabla \bar{n} \cdot \nabla V+\bar{n} \Delta V\right) \psi d x .
\end{aligned}
$$

Since, by assumption, $n_{k-1} \leq \bar{n}_{k-1}=\left(1-\tau\|\Delta V\|_{L^{\infty}\left(\mathbb{R}^{d)}\right)}\right) \bar{n}$, the first integral on the right-hand side is nonnegative. In order to estimate the second integral, we compute, on the set $\left\{x \in \mathbb{R}^{d}|R \leq| x \mid \leq m\right\}$,

$$
\begin{aligned}
I & :=\Delta f_{\varepsilon}(\bar{n})+\nabla \bar{n} \cdot \nabla V+\bar{n} \Delta V \\
& =\frac{\gamma}{|x|^{2}}\left(\gamma \bar{n} f^{\prime \prime}(\bar{n})+(\gamma+2-d) f^{\prime}(\bar{n})\right)+\frac{\varepsilon \gamma}{|x|^{2}}(\gamma+2-d) \bar{n}-\frac{\gamma x \cdot \nabla V}{|x|^{2}} \bar{n}+\bar{n} \Delta V \\
& =\frac{\alpha \gamma}{|x|^{2}}(\alpha \gamma+2-d) \bar{n}^{\alpha-1}+\frac{\varepsilon \gamma}{|x|^{2}}(\gamma+2-d) \bar{n}-\frac{\gamma x \cdot \nabla V}{|x|^{2}} \bar{n}+\bar{n} \Delta V .
\end{aligned}
$$


Using $0 \leq \bar{n} \leq c_{2} \exp \left(T\|\Delta V\|_{L^{\infty}\left(\mathbb{R}^{d}\right)}\right)$, we obtain

$$
\begin{aligned}
I \leq \bar{n}\left(\frac{\alpha \gamma}{R^{2}}|\alpha \gamma+2-d| c_{2}^{\alpha-1} e^{(\alpha-1) T\|\Delta V\|_{L^{\infty}\left(\mathbb{R}^{d}\right)}}\right. & +\frac{\gamma}{R^{2}}|\gamma+2-d| \\
& \left.-\frac{\gamma x \cdot \nabla V}{|x|^{2}}+\Delta V\right) .
\end{aligned}
$$

Here, we have employed the special form of $f(s)$. However, it is sufficient to assume that $s \mapsto \gamma s f^{\prime \prime}(s)+(\gamma+2-d) f^{\prime}(s)$ is nondecreasing for all $s>0$ (see Remark 2.1). Let $\delta=c_{0} \gamma-\|\Delta V\|_{L^{\infty}\left(\mathbb{R}^{d}\right)}>0$. Choosing $R \geq R_{0}$ $\left(R_{0}\right.$ is defined in $\left.(\mathrm{A} 2 \mathrm{~b})\right)$ such that

$$
R^{2} \geq \frac{\gamma}{\delta}\left(\alpha|\alpha \gamma+2-d| c_{2}^{\alpha-1} e^{(\alpha-1) T\|\Delta V\|_{L^{\infty}\left(\mathbb{R}^{d}\right)}}+|\gamma+2-d|\right),
$$

we obtain, taking into account assumption (A2),

$$
I \leq\left(\delta-\frac{2 \gamma x \cdot \nabla V}{|x|^{2}}+\Delta V\right) \bar{n} \leq\left(\delta-\gamma c_{0}+\|\Delta V\|_{L^{\infty}\left(\mathbb{R}^{d}\right)}\right) \bar{n}=0
$$

and finally $A \geq 0$, which proves the lemma.

Next, we prove that the discrete solution $n_{k}$ is bounded from above uniformly in $\tau$.

Corollary 3.1 (Uniform Bound from Above). Let (A2) hold and let $n_{k}$ be a weak solution to (12). Then

$$
n_{k} \leq \bar{n}_{k} \leq \bar{n} \quad \text { in } B_{m},
$$

where $\bar{n}$ is defined in (10).

Proof: The proof is by induction over $k$. Let $k=0$. Then, by assumption $(\mathrm{A} 2 \mathrm{~b})$,

$$
n_{0}=n_{I} \leq \bar{n}_{0} \quad \text { in } B_{m} .
$$

Assume that $n_{k-1} \leq \bar{n}_{k-1}$ in $B_{m}$. We have to prove that $n_{k} \leq \bar{n}_{k}$ in $B_{m}$. By Lemma 3.2 , the difference $n_{k}-\bar{n}_{k}$ solves the variational inequality

$$
\begin{aligned}
\frac{1}{\tau} \int_{B_{m}}\left(n_{k}\right. & \left.-\bar{n}_{k}\right) \psi d x-\frac{1}{\tau} \int_{B_{m}}\left(n_{k-1}-\bar{n}_{k}\right) \psi d x \\
& +\int_{B_{m}}\left(\nabla\left(f_{\varepsilon}\left(n_{k}\right)-f_{\varepsilon}\left(\bar{n}_{k}\right)\right)+\left(n_{k}-\bar{n}_{k}\right) \nabla V\right) \cdot \nabla \psi d x \leq 0
\end{aligned}
$$


for all $0 \leq \psi \in H^{1}\left(B_{m}\right)$. We take the test function $\psi=\operatorname{sign}_{\omega}^{+}\left(f_{\varepsilon}\left(n_{k}\right)-\right.$ $f_{\varepsilon}\left(\bar{n}_{k}\right)$ ), where $\operatorname{sign}_{\omega}^{+}$is defined as in Lemma 3.1. After similar computations as in the proof of Lemma 3.1, we arrive at

$$
\int_{B_{m}}\left(n_{k}-\bar{n}_{k}\right)^{+} d x=\int_{B_{m}}\left(n_{k-1}-\bar{n}_{k-1}\right)^{+} d x=0
$$

which implies that $n_{k} \leq \bar{n}_{k}$ in $B_{m}$.

\subsection{Proof of Theorem 2.1 under (A1) and (A2).}

In order to solve (7), we consider, for given $n_{k-1} \in L^{1}\left(\mathbb{R}^{d}\right) \cap L^{\infty}\left(\mathbb{R}^{d}\right)$ such that $\int_{B_{m}} n_{k-1} d x=\int_{B_{m}} n_{I} d x$, the regularized problem

$$
\begin{array}{ll}
\operatorname{div}\left(\nabla f_{\varepsilon}(n)+n^{+} \nabla V\right)=\frac{1}{\tau}\left(n-n_{k-1}\right) & \text { in } B_{m}, \\
\frac{\partial}{\partial \nu^{+}} f_{\varepsilon}(n)+n \frac{\partial V}{\partial \nu^{+}}=0 & \text { on } \partial B_{m},
\end{array}
$$

where $f_{\varepsilon}(s)=s^{\alpha}+\varepsilon s(s \geq 0), s^{-}=\min \{0, s\}(s \in \mathbb{R})$, and $\nu^{+}$is the outward normal unit vector on $\partial B_{m}$. Here, $\varepsilon$ and $m$ are positive parameters. Since the above boundary-value problem is uniformly elliptic, we obtain the existence of a unique solution $n=n_{\varepsilon, m, k} \in H^{2}\left(B_{m}\right)$ [16. Chapter 10, Theorem 2.2]. Employing the test function $\psi=n^{-} \in H^{1}\left(B_{m}\right)$ in the weak formulation of (19), we conclude immediately that $n \geq 0$ in $B_{m}$ (since $n^{+} \nabla V \cdot \nabla n^{-}=0$ ). Hence, we can write $n$ instead of $n^{+}$in (19). Moreover, using the test function $\psi=1$ in the weak formulation, it follows that $\int_{B_{m}} n d x=\int_{B_{m}} n_{k-1} d x=\int_{B_{m}} n_{I} d x$. Thus, we have an $L^{1}$ bound for $n$ uniformly in the parameters $\varepsilon$ and $m$.

The following steps are concerned with the limits $\varepsilon \rightarrow 0$ and $m \rightarrow \infty$. Let $m \geq R$, where $R>0$ is defined in (18).

First step: the limit $\varepsilon \rightarrow 0$ : A straightforward computation shows that $\bar{n}$, defined in (10), is a supersolution of the weak formulation of (19)-(20), namely

(21) $\int_{B_{m}} \nabla f_{\varepsilon}\left(n_{\varepsilon}\right) \cdot \nabla \psi d x=-\int_{B_{m}} n_{\varepsilon} \nabla V \cdot \nabla \psi d x-\frac{1}{\tau} \int_{B_{m}}\left(n_{\varepsilon}-n_{k-1}\right) \psi d x$ 
for appropriate test functions $\psi$. Taking now the test function $\psi=$ $f_{\varepsilon}\left(n_{\varepsilon}\right) \in H^{1}\left(B_{m}\right)$ in $(21)$, we obtain

$$
\begin{aligned}
\int_{B_{m}}\left|\nabla f_{\varepsilon}\left(n_{\varepsilon}\right)\right|^{2} d x= & -\int_{B_{m}} n_{\varepsilon} \nabla V \cdot \nabla f_{\varepsilon}\left(n_{\varepsilon}\right) d x \\
& -\frac{1}{\tau} \int_{B_{m}}\left(n_{\varepsilon}-n_{k-1}\right) f_{\varepsilon}\left(n_{\varepsilon}\right) d x \\
\leq & \int_{B_{m}} \Delta V F_{\varepsilon}\left(n_{\varepsilon}\right) d x-\frac{1}{\tau} \int_{B_{m}}\left(n_{\varepsilon}-n_{k-1}\right) f_{\varepsilon}\left(n_{\varepsilon}\right) d x \\
\leq & C\|\Delta V\|_{L^{\infty}\left(\mathbb{R}^{d}\right)} \int_{B_{m}} F_{\varepsilon}\left(n_{\varepsilon}\right) d x-\frac{1}{\tau} \int_{B_{m}} n_{\varepsilon}^{\alpha+1} d x \\
& +\frac{1}{\tau} \int_{B_{m}} n_{k-1} f_{\varepsilon}\left(n_{\varepsilon}\right) d x
\end{aligned}
$$

where the function $F_{\varepsilon}$ is defined as $F_{\varepsilon}^{\prime}(s)=s f_{\varepsilon}^{\prime}(s)$ with $F_{\varepsilon}(0)=0$ and we have used that $x \cdot \nabla V>0$ due to $(\mathrm{A} 2 \mathrm{~b})$ on $\partial B_{m}$. Since $F_{\varepsilon}(s) / s$ is a uniformly in $\varepsilon$ bounded function on intervals of the form $[0, B]$ with $B>0$ and using the uniformly in $\varepsilon$ upper bound for $n_{\varepsilon}$ given by (8), we conclude that $\left\|\nabla f_{\varepsilon}\left(n_{\varepsilon}\right)\right\|_{L^{2}\left(B_{m}\right)} \leq c$, where $c>0$ is independent of $\varepsilon$ and $m$. Therefore,

$$
\left\|f_{\varepsilon}\left(n_{\varepsilon}\right)\right\|_{H^{1}\left(B_{m}\right)} \leq c
$$

where $c>0$ does not depend on $\varepsilon$. Thus, there exists a subsequence of $\left(n_{\varepsilon}\right)$ (not relabeled) such that, as $\varepsilon \rightarrow 0$,

$$
\begin{array}{r}
n_{\varepsilon} \rightarrow n \quad \text { weakly in } L^{2}\left(B_{m}\right) \text { and weakly* in } L^{\infty}\left(B_{m}\right), \\
f_{\varepsilon}\left(n_{\varepsilon}\right) \rightarrow w \quad \text { weakly in } H^{1}\left(B_{m}\right) \text { and strongly in } L^{2}\left(B_{m}\right) .
\end{array}
$$

Since strong $L^{2}$ convergence gives pointwise convergence a.e. for a subsequence and $f_{\varepsilon}(s)=f(s)+\varepsilon s$ is increasing, we obtain

$$
n_{\varepsilon}=f^{-1}\left(f_{\varepsilon}\left(n_{\varepsilon}\right)-\varepsilon n_{\varepsilon}\right) \rightarrow f^{-1}(w) \text { pointwise a.e., }
$$

and we conclude that $f^{-1}(w)=n$ and $w=f(n)$. Passing to the limit $\varepsilon \rightarrow 0$ in (21) then gives

$$
\int_{B_{m}}(\nabla f(n)+n \nabla V) \cdot \nabla \psi d x=-\frac{1}{\tau} \int_{B_{m}}\left(n-n_{k-1}\right) \psi d x
$$

for test functions $\psi \in H^{1}\left(\mathbb{R}^{d}\right)$. 
Second step: the limit $m \rightarrow \infty$ : Let $n_{m} \in L^{\infty}\left(B_{m}\right)$ with $f\left(n_{m}\right) \in$ $H^{1}\left(B_{m}\right)$ be a solution to $(24)$ with $n=n_{m}$. We claim that there is a constant $c>0$ such that for all $m,\left\|f\left(n_{m}\right)\right\|_{H^{1}\left(B_{m}\right)} \leq c$. Indeed, using again $(22), \nabla f\left(n_{m}\right)$ is bounded in $L^{2}\left(B_{m}\right)$ uniformly in $m$ since

$$
\left\|f\left(n_{m}\right)\right\|_{L^{2}\left(B_{m}\right)}^{2}=\int_{B_{m}} n_{m}^{2 \alpha} d x \leq\|\bar{n}\|_{L^{\infty}\left(\mathbb{R}^{d}\right)}^{2 \alpha-1} \int_{\mathbb{R}^{d}} n_{m} d x \leq c,
$$

and $c>0$ does not depend on $m$. Thus, we apply a standard Cantor diagonal selection argument to derive the convergence to a solution of the discrete problem bounded by $\bar{n}$ as desired, finishing the proof of Theorem 2.1.

\subsection{Proof of Theorem 2.2 under (A1) and (A2).}

We define the piecewise constant function in time $n^{(N)}(x, t)=n_{k}(x)$ if $x \in B_{m}$ for all fixed $m>0, t \in\left(t_{k-1}, t_{k}\right]$, and the shift operator $\left(\sigma_{N} n^{(N)}\right)(\cdot, t)=n_{k-1}$ for $t \in\left(t_{k-1}, t_{k}\right]$. By Theorem 2.1, $n^{(N)}$ solves the problem

(25) $\frac{1}{\tau}\left(n^{(N)}-\sigma_{N} n^{(N)}\right)=\operatorname{div}\left(\nabla f\left(n^{(N)}\right)+n^{(N)} \nabla V\right) \quad$ in $B_{m}, 0<t<T$,

subject to no-flux boundary conditions. In order to derive an estimate for the discrete time derivative, we compute

$$
\begin{aligned}
& \tau^{-2}\left\|n^{(N)}-\sigma_{N} n^{(N)}\right\|_{L^{2}\left(0, T ;\left(H^{1}\left(B_{m}\right)\right)^{*}\right)}^{2} \\
& \quad \leq \sup _{\|\psi\|_{H^{1}\left(B_{m}\right)}=1} \int_{0}^{T}\left(\int_{B_{m}}\left(\nabla f\left(n^{(N)}\right)+n^{(N)} \nabla V\right) \cdot \nabla \psi d x\right)^{2} d t \\
& \leq\left\|\nabla f\left(n^{(N)}\right)\right\|_{L^{2}\left(0, T ; L^{2}\left(B_{m}\right)\right)}^{2}+\|\bar{n} \mid \nabla V\|_{L^{2}\left(0, T ; L^{2}\left(B_{m}\right)\right)}^{2} \leq c,
\end{aligned}
$$

where $c>0$ is independent of $m$ and $N$ due to (A2) since $\gamma>(d+2) / 2$ and $\nabla V$ grows at most linearly at infinity. Thus, for a subsequence (not relabeled), as $N \rightarrow \infty$ or $\tau \rightarrow 0$,

$$
\frac{1}{\tau}\left(n^{(N)}-\sigma_{N} n^{(N)}\right) \rightarrow \partial_{t} n \quad \text { weakly in } L^{2}\left(0, T ;\left(H^{1}\left(B_{m}\right)\right)^{*}\right) .
$$

The proof of Theorem 2.1 provides the following a priori estimates:

$$
\left\|n^{(N)}\right\|_{L^{\infty}\left(B_{m}\right)} \leq c, \quad\left\|f\left(n^{(N)}\right)\right\|_{L^{2}\left(0, T, H^{1}\left(B_{m}\right)\right.} \leq c,
$$

where the constant $c>0$ does not depend on $\tau, N$ or $m$. Thus, as $N \rightarrow \infty$, for a subsequence which is not relabeled,

$$
f\left(n^{(N)}\right)=\left(n^{(N)}\right)^{\alpha} \rightarrow w \quad \text { weakly in } L^{2}\left(0, T ; H^{1}\left(B_{m}\right)\right) .
$$


Since $x \mapsto x^{1 / \alpha}$ is Hölder continuous with exponent $1 / \alpha$, we conclude from the $H^{1}$ estimate for $n^{(N)}$ that (see [9, p. 141])

$$
\left\|n^{(N)}\right\|_{W^{1 / \alpha, 2 \alpha}\left(B_{m}\right)} \leq c\left\|\left(n^{(N)}\right)^{\alpha}\right\|_{H^{1}\left(B_{m}\right)} \leq c,
$$

and the constant $c>0$ depends on $\alpha$ but not on $m$ or $N$. Since the embedding $W^{s, 2 \alpha}\left(B_{m}\right) \hookrightarrow W^{1 / \alpha, 2 \alpha}\left(B_{m}\right)$ is compact for all $s<1 / \alpha$, we can apply Aubin's lemma [22] to obtain the existence of a subsequence (not relabeled) of $n^{(N)}$ such that, as $N \rightarrow \infty$,

$$
n^{(N)} \rightarrow n \text { strongly in } L^{2}\left(0, T ; W^{s, 2 \alpha}\left(B_{m}\right)\right)
$$

and $n^{(N)} \rightarrow n$ pointwise a.e. In particular, $\left(n^{(N)}\right)^{\alpha} \rightarrow n^{\alpha}$ pointwise a.e. and $w=n^{\alpha}$.

The convergence results (26)-(28) allow to pass to the limit $N \rightarrow \infty$ in the weak formulation of (25), leading to

$\int_{0}^{T}\left\langle\partial_{t} n, \psi\right\rangle_{\left(H^{1}\left(B_{m}\right)\right)^{*}, H^{1}\left(B_{m}\right)} d t+\int_{0}^{T} \int_{B_{m}}\left(\nabla\left(n^{\alpha}\right)+n \nabla V\right) \cdot \nabla \psi d x d t=0$, for all $\psi \in H^{1}\left(B_{m}\right)$. Since the above estimates are uniform in $m$, we can pass to the limit $m \rightarrow \infty$ and obtain a weak solution to (9).

\subsection{Existence of solutions under (A1) and (A2').}

We show Theorems 2.1 and 2.2 by replacing assumption (A2) by (A2'). For given $n_{k-1} \in H^{1}\left(B_{m}\right)$, consider the approximated problem (19)-(20) and assume that (A1) and (A2') holds. We set $h_{\varepsilon}(s)=h(s)+\varepsilon \log s$, $s>0$, where $h(s)$ is defined in (5). Notice that $h_{\varepsilon}^{-1}$ exists on $\mathbb{R}$, $\lim _{s \rightarrow \infty} h_{\varepsilon}^{-1}(s)=\infty$, and $h_{\varepsilon}^{-1}(s)>0$ for all $s \in \mathbb{R}$.

Lemma 3.3 (Supersolution for (A2')). Let $n_{\varepsilon, k} \in H^{1}\left(B_{m}\right)$ be a solution of (19)-(20) and set $\bar{n}_{\varepsilon}=h_{\varepsilon}^{-1}(K-V)$, where $K \in \mathbb{R}$ is such that $\bar{n}_{\varepsilon} \geq n_{I}$ in $B_{m}$. Let $n_{\varepsilon, k-1} \leq \bar{n}_{\varepsilon}$ in $B_{m}$. Then $n_{\varepsilon, k} \leq \bar{n}_{\varepsilon}$ in $B_{m}$.

The lemma can be shown similarly as Lemma 3.1 , stated in $B_{m}$, since $\nabla f_{\varepsilon}\left(\bar{n}_{\varepsilon}\right)+\bar{n}_{\varepsilon} \nabla V=\bar{n}_{\varepsilon} \nabla\left(h\left(\bar{n}_{\varepsilon}\right)+V\right)=0$ in $B_{m}$.

Now, the proof of Theorem 2.1 is similar to the proof under assumption (A2), with the following change in the first step of the proof. The upper bound $\bar{n}_{\varepsilon}$ is chosen to converge pointwise a.e., in fact uniformly in compact sets, to $\bar{n}=h^{-1}(K-V)$ as $\varepsilon \rightarrow 0$ such that $\bar{n}_{\varepsilon} \geq n_{I}$ for small $\varepsilon$ and $\bar{n} \geq n_{I}$. Therefore, in view of the uniform $H^{1}$ estimates for $f_{\varepsilon}\left(n_{\varepsilon, k}\right)$, for a subsequence,

$$
\begin{aligned}
n_{\varepsilon, k} \rightarrow n_{k} \leq \bar{n} & \text { strongly in } L^{2}\left(B_{m}\right), \\
f_{\varepsilon}\left(n_{\varepsilon, k}\right) \rightarrow f\left(n_{k}\right) & \text { weakly in } H^{1}\left(B_{m}\right) .
\end{aligned}
$$


428

J. A. Carrillo, M. P. Gualdani, A. Jüngel

These limits show that $n_{k}$ is a solution of (24). Finally, the proof of Theorem 2.2 is analogous to the proof in the previous section.

4. Proof of Theorem 2.4

First, we notice that the weak formulation (9) is valid for all functions in $L^{2}\left(0, T ; H^{1}\left(\mathbb{R}^{d}\right)\right)$ since $C_{0}^{\infty}\left(\mathbb{R}^{d}\right)$ is dense in $H^{1}\left(\mathbb{R}^{d}\right)$. Let $n_{1}$ and $n_{2}$ be two weak solutions to (1)-(2) in the sense of Theorem 2.2 with the same initial data, let $t \in(0, T)$, and let $\psi(\cdot, t) \in H^{1}\left(\mathbb{R}^{d}\right)$ be the unique solution to

$$
\Delta \psi=-\left(n_{1}-n_{2}\right)(\cdot, t) \quad \text { in } \mathbb{R}^{d} .
$$

Then $\psi \in L^{2}\left(0, T ; H^{1}\left(\mathbb{R}^{d}\right)\right)$ and, since $n_{1}$ and $n_{2}$ satisfy the same initial data, $\psi(\cdot, 0)=0$. Thus, we can employ $\psi$ as a test function in the difference of the weak formulations for $n_{1}$ and $n_{2}$,

$$
\begin{aligned}
& \int_{0}^{t}\left\langle\partial_{t}\left(n_{1}-n_{2}\right), \psi\right\rangle_{H^{-1}, H^{1}} d t \\
& \quad=-\int_{0}^{t} \int_{\mathbb{R}^{d}}\left(\nabla\left(f\left(n_{1}\right)-f\left(n_{2}\right)\right)+\left(n_{1}-n_{2}\right) \nabla V\right) \cdot \nabla \psi d x d t
\end{aligned}
$$

The left-hand side can be written as

$$
-\int_{0}^{t}\left\langle\partial_{t} \Delta \psi, \psi\right\rangle_{H^{-1}, H^{1}} d t=\int_{0}^{t} \int_{\mathbb{R}^{d}} \partial_{t} \nabla \psi \cdot \nabla \psi d x d t=\frac{1}{2} \int_{\mathbb{R}^{d}}|\nabla \psi(\cdot, t)|^{2} d x .
$$

The first term on the right-hand side of (29) becomes, after integrating by parts,

$$
\int_{0}^{t} \int_{\mathbb{R}^{d}}\left(f\left(n_{1}\right)-f\left(n_{2}\right)\right) \Delta \psi d x d t=-\int_{0}^{t} \int_{\mathbb{R}^{d}}\left(f\left(n_{1}\right)-f\left(n_{2}\right)\right)\left(n_{1}-n_{2}\right) d x d t \leq 0,
$$

since $f$ is nondecreasing. For the drift term, we also integrate by parts:

$$
\begin{aligned}
\int_{0}^{t} \int_{\mathbb{R}^{d}}( & \left.n_{1}-n_{2}\right) \nabla V \cdot \nabla \psi d x d t=-\int_{0}^{t} \int_{\mathbb{R}^{d}} \Delta \psi \nabla V \cdot \nabla \psi d x d t \\
= & \int_{0}^{t} \int_{\mathbb{R}^{d}} \nabla \psi \cdot \nabla(\nabla V \cdot \nabla \psi) d x d t \\
= & \int_{0}^{t} \int_{\mathbb{R}^{d}}(\nabla \psi)^{\top} \operatorname{Hess}(V)(\nabla \psi)-\frac{1}{2} \int_{0}^{t} \int_{\mathbb{R}^{d}} \Delta V|\nabla \psi|^{2} d x d t \\
\leq & \left(1+\frac{d}{2}\right)\|V\|_{W^{2, \infty}\left(\mathbb{R}^{d}\right)} \int_{0}^{t} \int_{\mathbb{R}^{d}}|\nabla \psi|^{2} d x d t,
\end{aligned}
$$


where $\operatorname{Hess}(V)$ denotes the Hessian of $V$. We obtain from (29)

$$
\frac{1}{2} \int_{\mathbb{R}^{d}}|\nabla \psi(\cdot, t)|^{2} d x \leq\left(1+\frac{d}{2}\right)\|V\|_{W^{2, \infty}\left(\mathbb{R}^{d}\right)} \int_{0}^{t} \int_{\mathbb{R}^{d}}|\nabla \psi|^{2} d x d t .
$$

Gronwall's lemma shows that $\nabla \psi=0$ and thus, $n_{1}-n_{2}=0$, ending the proof.

Remark 4.1. If the diffusion is nondegenerate, it is enough to assume that $\Delta V \in L^{\infty}\left(\mathbb{R}^{d}\right)$. For instance, if $f(s)=s$, we can take $n_{1}-n_{2}$ as a test function in the difference of the equations satisfied by the two solutions $n_{1}$ and $n_{2}$. Then the drift term can be estimated by

$$
\begin{aligned}
\int_{0}^{t} \int_{\mathbb{R}^{d}} & \nabla\left(n_{1}-n_{2}\right) \nabla V \cdot \nabla\left(n_{1}-n_{2}\right) d x d t \\
= & \frac{1}{2} \int_{0}^{t} \int_{\mathbb{R}^{d}} \nabla V \cdot \nabla\left(\left(n_{1}-n_{2}\right)^{2}\right) d x d t \\
= & -\frac{1}{2} \int_{0}^{t} \int_{\mathbb{R}^{d}} \Delta V\left(n_{1}-n_{2}\right)^{2} d x d t,
\end{aligned}
$$

and a Gronwall argument gives the conclusion. If $f$ is not linear but still nondegenerate (i.e. $f(0) \neq 0$ ) then one can try to employ the dual method like in [10].

\section{Proof of the entropy decay}

In this section we prove Theorem 2.3. Let $n_{k}=n_{k, m, \varepsilon} \in L^{\infty}\left(B_{m}\right) \cap$ $H^{1}\left(B_{m}\right)$ with $f\left(n_{k}\right) \in H^{1}\left(B_{m}\right)$ be a solution to the uniformly elliptic problem

(30) $\int_{B_{m}} \nabla f_{\varepsilon}\left(n_{k}\right) \cdot \nabla \psi d x=-\int_{B_{m}} n_{k} \nabla V \cdot \nabla \psi d x-\frac{1}{\tau} \int_{B_{m}}\left(n_{k}-n_{k-1}\right) \psi d x$,

for all test functions $\psi \in H^{1}\left(B_{m}\right)$ such that $n_{0}=\chi_{m} n_{I}$, where $\chi_{m}(x)=$ $\chi(x / m)$ is the cut-off function with $\chi \in C_{0}^{\infty}\left(\mathbb{R}^{d}\right)$ satisfying $\chi(x)=1$ for $|x| \leq 1, \chi(x)=0$ for $|x| \geq 2$, and $0<\chi(x)<1$ for $1<|x|<2$. Set $h_{\varepsilon}(s)=h(s)+\varepsilon \log s, H_{\varepsilon}^{\prime}(s)=h_{\varepsilon}(s)$ for $s>0$, and

$$
E_{\varepsilon}\left(n_{k}\right)=\int_{B_{m}}\left(H_{\varepsilon}\left(n_{k}\right)+n_{k} V\right) d x
$$


Since $H_{\varepsilon}$ is convex, it follows

$$
\begin{aligned}
E_{\varepsilon}\left(n_{k}\right)-E_{\varepsilon}\left(n_{k-1}\right) & =\int_{B_{m}}\left(H_{\varepsilon}\left(n_{k}\right)-H_{\varepsilon}\left(n_{k-1}\right)+\left(n_{k}-n_{k-1}\right) V\right) d x \\
& \leq \int_{B_{m}}\left(H_{\varepsilon}^{\prime}\left(n_{k}\right)+V\right)\left(n_{k}-n_{k-1}\right) d x .
\end{aligned}
$$

Employing the test function $\psi=h_{\varepsilon}\left(n_{k}\right)+V \in H^{1}\left(B_{m}\right)$ in (30) gives

$$
\frac{1}{\tau} \int_{B_{m}}\left(n_{k}-n_{k-1}\right)\left(h_{\varepsilon}\left(n_{k}\right)+V\right) d x=-\int_{B_{m}} n_{k}\left|\nabla\left(h_{\varepsilon}\left(n_{k}\right)+V\right)\right|^{2} d x .
$$

Therefore, by [6, Theorem 17],

$$
\begin{aligned}
E_{\varepsilon}\left(n_{k}\right)-E_{\varepsilon}\left(n_{k-1}\right) & \leq-\tau \int_{B_{m}} n_{k}\left|\nabla\left(h_{\varepsilon}\left(n_{k}\right)+V\right)\right|^{2} d x \\
& \leq-2 \lambda \tau\left(E_{\varepsilon}\left(n_{k}\right)-E_{\varepsilon}\left(n_{\infty, \varepsilon, m}\right)\right)
\end{aligned}
$$

and

$$
\begin{aligned}
E_{\varepsilon}\left(n_{k}\right)-E_{\varepsilon}\left(n_{\infty, \varepsilon, m}\right) & \leq(1+2 \lambda \tau)^{-1}\left(E_{\varepsilon}\left(n_{k-1}\right)-E_{\varepsilon}\left(n_{\infty, \varepsilon, m}\right)\right) \\
& \leq(1+2 \lambda \tau)^{-k}\left(E_{\varepsilon}\left(n_{0}\right)-E_{\varepsilon}\left(n_{\infty, \varepsilon, m}\right)\right) .
\end{aligned}
$$

Now, we wish to perform the limits $\varepsilon \rightarrow 0$ and $m \rightarrow \infty$. We write $n_{\varepsilon, k}$ instead of $n_{k}$. The proof is exactly as in the previous section, showing that, as $\varepsilon \rightarrow 0$, up to a subsequence,

$$
\begin{aligned}
& n_{\varepsilon, k} \rightarrow n_{k} \quad \text { strongly in } L^{2}\left(B_{m}\right), \\
& f_{\varepsilon}\left(n_{\varepsilon, k}\right) \rightarrow f\left(n_{k}\right) \quad \text { weakly in } H^{1}\left(B_{m}\right) .
\end{aligned}
$$

In view of the uniform $L^{\infty}$ bound for $n_{\varepsilon, k}$ and the convergence a.e. of $n_{\varepsilon, k} \rightarrow n_{k}$, Lebesgue's theorem implies

$$
E_{\varepsilon}\left(n_{\varepsilon, k}\right) \rightarrow E\left(n_{k}\right) \quad \text { as } k \rightarrow \infty .
$$

The limits $E_{\varepsilon}\left(n_{0}\right) \rightarrow E\left(n_{0}\right)$ and $E_{\varepsilon}\left(n_{\infty, \varepsilon, m}\right) \rightarrow E\left(n_{\infty, m}\right)$ as $\varepsilon \rightarrow 0$ are easy consequences of the convergence in compact sets of $H_{\varepsilon}\left(n_{0}\right) \rightarrow H\left(n_{0}\right)$ and $H_{\varepsilon}\left(n_{\infty, \varepsilon, m}\right) \rightarrow H\left(n_{\infty, m}\right)$. Thus, we can pass to the limit $\varepsilon \rightarrow 0$ in (31), giving

$$
E\left(n_{k, m}\right)-E\left(n_{\infty, m}\right) \leq(1+2 \lambda \tau)^{-k}\left(E\left(n_{I}\right)-E\left(n_{\infty, m}\right)\right) .
$$

The proof of $E\left(n_{k, m}\right) \rightarrow E\left(n_{k}\right)$ as $m \rightarrow \infty$ can be performed analogously to the arguments in the previous section by using the control at infinity given by $\bar{n}$ in the case of (A2) or the compact support of the solutions in the case of (A2') to apply Lebesgue's theorem. The proof concludes by showing $E\left(n_{\infty, m}\right) \rightarrow E\left(n_{\infty}\right)$ as $m \rightarrow \infty$ by direct inspection since 
in fact $n_{\infty, m}=n_{\infty}$ for sufficiently large $m$ due to the compact support of $n_{\infty}$.

Acknowledgements. JAC acknowledges the support from DGI-MEC (Spain) FEDER-project MTM2005-08024 and 2005SGR00611. AJ acknowledges partial support from the Deutsche Forschungsgemeinschaft, grants JU359/5 and JU359/7 and from the Wissenschaftskolleg "Differential Equations", funded by the Fonds zur Förderung der wissenschaftlichen Forschung. This research is part of the ESF program "Global and geometrical aspects of nonlinear partial differential equations (GLOBAL)". JAC and AJ acknowledge partial support of the Acciones Integradas Program HU2006-0025.

\section{References}

[1] L. Ambrosio, N. Gigli, and G. Savaré, "Gradient flows in metric spaces and in the space of probability measures", Lectures in Mathematics ETH Zürich, Birkhäuser Verlag, Basel, 2005.

[2] P. Bénilan and M. G. Crandall, The continuous dependence on $\varphi$ of solutions of $u_{t}-\Delta \varphi(u)=0$, Indiana Univ. Math. J. 30(2) (1981), 161-177.

[3] M. Bertsch And D. A. Hilhorst, A density dependent diffusion equation in population dynamics: stabilization to equilibrium, SIAM J. Math. Anal. 17(4) (1986), 863-883.

[4] P. Biler, J. Dolbeault, and P. A. Markowich, Large time asymptotics of nonlinear drift-diffusion systems with Poisson coupling, The Sixteenth International Conference on Transport Theory, Part II (Atlanta, GA, 1999), Transport Theory Statist. Phys. 30(4-6) (2001), 521-536.

[5] J. A. Carrillo, M. Di Francesco, and M. P. Gualdani, Semidiscretization and long-time asymptotics of nonlinear diffusion equations, Commun. Math. Sci. 5, Supplement (2007), 21-53.

[6] J. A. Carrillo, A. Jüngel, P. A. Markowich, G. Toscani, AND A. Unterreiter, Entropy dissipation methods for degenerate parabolic problems and generalized Sobolev inequalities, Monatsh. Math. 133(1) (2001), 1-82.

[7] J. A. Carrillo, R. J. McCann, and C. Villani, Kinetic equilibration rates for granular media and related equations: entropy dissipation and mass transportation estimates, Rev. Mat. Iberoamericana 19(3) (2003), 971-1018. 
[8] P.-H. Chavanis, Generalized thermodynamics and Fokker-Planck equations: Applications to stellar dynamics and two-dynamical turbulence, Phys. Rev. E 68 (2003), 036108.

[9] G. Chavent and J. Jaffre, "Mathematical Models and Finite Elements for Reservoir Simulation-Single Phase, Multiphase and Multicomponent Flows through Porous Media", Studies in Mathematics and its Applications 17, North-Holland, Amsterdam, 1986.

[10] J. I. Díaz, G. Galiano, and A. Jüngel, On a quasilinear degenerate system arising in semiconductors theory. I. Existence and uniqueness of solutions, Nonlinear Anal. Real World Appl. 2(3) (2001), 305-336.

[11] W. FAng AND K. Ito, Solutions to a nonlinear drift-diffusion model for semiconductors, Electron. J. Differential Equations 1999(15) (1999), 1-38 (electronic).

[12] J. Fiestas, R. Spurzem, and E. Kim, 2D Fokker-Planck models of rotating clusters, Monthly Notices Roy. Astronom. Soc. (to appear).

[13] T. D. Frank, "Nonlinear Fokker-Planck equations", Fundamentals and applications, Springer Series in Synergetics, Springer-Verlag, Berlin, 2005.

[14] A. JÜNGEL, On the existence and uniqueness of transient solutions of a degenerate nonlinear drift-diffusion model for semiconductors, Math. Models Methods Appl. Sci. 4(5) (1994), 677-703.

[15] A. JÜNGEL, "Quasi-hydrodynamic semiconductor equations", Progress in Nonlinear Differential Equations and their Applications 41, Birkhäuser Verlag, Basel, 2001.

[16] O. A. Ladyzhenskaya and N. N. Ural'tseva, "Linear and quasilinear elliptic equations", Translated from the Russian by Scripta Technica, Inc. Translation editor: Leon Ehrenpreis, Academic Press, New York-London, 1968.

[17] P. A. Markowich, C. A. Ringhofer, and C. Schmeiser, "Semiconductor equations", Springer-Verlag, Vienna, 1990.

[18] J. NiETO, Hydrodynamical limit for a drift-diffusion system modeling large-population dynamics, J. Math. Anal. Appl. 291(2) (2004), $716-726$.

[19] F. Отто, The geometry of dissipative evolution equations: the porous medium equation, Comm. Partial Differential Equations 26(1-2) (2001), 101-174.

[20] N. Rostoker And M. N. Rosenbluth, Fokker-Planck equation for a plasma with a constant magnetic field, J. Nucl. Energy. Part C: Plasma Physics 2 (1961), 195-205. 
[21] J. Rulla, Weak solutions to Stefan problems with prescribed convection, SIAM J. Math. Anal. 18(6) (1987), 1784-1800.

[22] J. Simon, Compact sets in the space $L^{p}(0, T ; B)$, Ann. Mat. Pura Appl. (4) 146 (1987), 65-96.

[23] J. L. VÁzQuez, Asymptotic beahviour for the porous medium equation posed in the whole space, Dedicated to Philippe Bénilan, J. Evol. Equ. 3(1) (2003), 67-118.

[24] J. L. VÁzQuez, "The porous medium equation", Mathematical theory, Oxford Mathematical Monographs, The Clarendon Press, Oxford University Press, Oxford, 2007.

[25] C. Villani, "Topics in optimal transportation", Graduate Studies in Mathematics 58, American Mathematical Society, Providence, RI, 2003.

\section{J. A. Carrillo:}

ICREA (Institució Catalana de Recerca i Estudis Avançats) and

Departament de Matemàtiques

Universitat Autònoma de Barcelona

08193 Bellaterra (Barcelona)

Spain

E-mail address: carrillo@mat.uab.es

M. P. Gualdani:

Department of Mathematics

University of Texas at Austin

78712 Austin

USA

E-mail address: gualdani@math.utexas.edu

A. Jüngel:

Institut für Analysis und Scientific Computing

Technische Universität Wien

Wiedner Hauptstr. 8-10

1040 Wien

Austria

E-mail address: juengel@anum.tuwien.ac.at

Primera versió rebuda el 12 de juny de 2007, darrera versió rebuda el 10 d'octubre de 2007. 